Relations industrielles

Industrial Relations

\title{
Weiss, David S., traduction de Jean Boivin, Franchir le mur des conflits
}

\section{Michel Grant}

Volume 55, numéro 1, 2000

URI : https://id.erudit.org/iderudit/051298ar

DOI : https://doi.org/10.7202/051298ar

Aller au sommaire du numéro

Éditeur(s)

Département des relations industrielles de l'Université Laval

ISSN

0034-379X (imprimé)

1703-8138 (numérique)

Découvrir la revue

Citer ce compte rendu

Grant, M. (2000). Compte rendu de [Weiss, David S., traduction de Jean Boivin, Franchir le mur des conflits]. Relations industrielles / Industrial Relations, 55(1), 176-179. https://doi.org/10.7202/051298ar

Tous droits réservés (C Département des relations industrielles de l'Université Laval, 2000
Ce document est protégé par la loi sur le droit d'auteur. L'utilisation des services d'Érudit (y compris la reproduction) est assujettie à sa politique d'utilisation que vous pouvez consulter en ligne.

https://apropos.erudit.org/fr/usagers/politique-dutilisation/ 
however, that in order to answer the questions that the volume commences with more than an extensive overview of developments in key corporations is called for. There are, for example, debates around the authenticity of a new knowledge based economy and the new training and skill sets that may (or may not) accompany it that need to be canvassed. Additionally, the issues of trade union "buy-in" to such agendas as multiskilling, team organization, and continu- ous improvement require a nuanced multi-dimensional and critical analysis that is often missing in this volume. The extent of corporate downsizing, as depressingly recounted in chapter after chapter, demands nothing less.

BOB RUSSELL School of Industrial Relations Griffith University Brisbane, Australia

\section{Franchir le mur des conflits}

par David S. WEISS, traduction de Jean BoIVIN, Québec : Les Presses de l'Université Laval, 1999, 277 p., ISBN 2-7637-7650-7.

Ce volume présente la traduction française réalisée par le professeur Jean Boivin, du Département des relations industrielles de l'Université Laval, du volume Beyond the Walls of Conflict de David S. Weiss publié en $1996 \mathrm{chez}$ Irwin.

Avant d'aborder le texte de David Weiss, il faut souligner que Jean Boivin ne s'est pas limité à un travail de traduction. La préface qu'il signe met en relief la spécificité et l'utilité de la contribution de David Weiss. Boivin rappelle en effet que ce dernier est un praticien et qu'à ce titre son expérience et son approche sont marquées par le réalisme à l'égard des attentes et des conditions d'implantation de ce qu'on appelle le plus souvent la négociation « raisonnée », mais qu'il qualifie de l'appellation plus appropriée de négociation basée sur les intérêts. Il s'agit bien d'un nouveau mode de règlement des différends et non pas d'une nouvelle mode. En effet, ce type de négociation constitue essentiellement une démarche axée sur la résolution de problèmes.

En plus de la préface du traducteur, la version française comporte une première annexe où l'on retrouve deux conventions collectives pour lesquelles les parties ont utilisé la nouvelle approche, soit une convention chez Bell Canada et une autre à Abitibi-Price d'Alma. Une seconde annexe présente deux études de cas. Le premier cas porte sur une intervention de l'auteur dans une usine de Northern Telecom à North York où la négociation basée sur les intérêts a été utilisée pour permettre la survie de cet établissement dont on avait annoncé la fermeture. La présentation décrit le potentiel mais aussi les contraintes et limites de la nouvelle approche. Le second cas, préparé par Jean Boivin, porte sur la transformation des relations du travail à Hydro-Québec mais ne traite pas spécifiquement d'une expérience de négociation basée sur les intérêts. Il s'agit surtout des conditions et des écueils vécus par les parties dans leur cheminement vers la construction et, peut-être, la fin d'un partenariat patronalsyndical.

Weiss s'inspire des principes et de l'approche développés par Roger Fisher et William Ury dans leur ouvrage classique Getting to Yes (traduit sous le titre Comment réussir une négociation aux Éditions du Seuil) mais va plus loin dans la mise en œuvre et l'adaptation de ces principes à la négociation des conventions collectives et des autres problèmes surgissant lors des relations patronalessyndicales. En effet, Weiss opérationnalise et articule de façon concrète une 
méthode adaptée au contexte conflictuel des relations du travail, tout en tenant compte des caractéristiques des acteurs institutionnels et individuels de même que des règles et des contraintes dans lesquelles se déploie le processus de négociation.

La notion de pouvoir et de conflit est largement évacuée par Fisher et Ury dont le modèle implique que les acteurs peuvent ultimement rompre la négociation. Or, la relation patronale-syndicale est une relation permanente et, en un sens, captive puisque les parties sont « condamnées » à s'entendre ou du moins à vivre ensemble en fonction de règles résultant de leurs échanges.

Toute négociation implique que les parties ont des intérêts, des droits et un certain pouvoir. L'expression des intérêts vise à dépasser les limites de la négociation positionnelle et à cerner le problème à l'origine d'une revendication. Les droits renvoient à ce qu'une partie voit le plus souvent comme une obligation de l'autre à son égard (ex., jurisprudence, loi, coutume, etc.). Le pouvoir renvoie aux méthodes basées sur la force, la défense de notre position (d'où le terme négociation positionnelle), le recours aux attaques personnelles, les tactiques psychologiques, etc. La négociation traditionnelle ou conflictuelle se différencie de la négociation basée sur les intérêts par l'importance du pouvoir et de son utilisation. Combien de fois entend-on l'expression « rapport de forces » dans les débats sur cette question! Weiss s'inspire évidemment de Fisher et Ury lorsqu'il propose aux parties d'identifier leurs intérêts à partir de la reconnaissance d'un problème qu'on cherche à résoudre par la négociation, et plus spécifiquement par le recours à la technique du remue-méninges comme génératrice de solutions créatrices et imaginatives. Alors que le pouvoir occupe la première place en négociation conflictuelle, on peut dire que le succès de la négociation basée sur les intérêts dépend du degré de non- utilisation de ce pouvoir dans le cheminement conduisant à une solution négociée et mutuellement bénéfique.

Un des grands mérites de cet ouvrage est qu'il présente à la fois des concepts et des pratiques. Ce n'est pas un livre de recettes. En aucun moment l'auteur ne prétend offrir des solutions magiques et faciles. Il offre un certain cadre de référence intellectuel et des moyens concrets pour actualiser le modèle de négociation privilégié. L'expérience de Weiss comme formateur et intervenant dans des dossiers de négociation lui a permis d'identifier les défis auxquels se confronte l'implantation de la négociation raisonnée. La condition initiale pour instaurer ce nouveau type de négociation renvoie à l'existence d'un lien de confiance. Il s'agit là d'un préalable à l'identification de solutions imaginatives et mutuellement bénéfiques.

Le cycle de la négociation basée sur les intérêts comporte cinq phases selon Weiss : planifier la négociation, commencer la négociation, résoudre les problèmes sur la base des intérêts, parvenir à une entente et vivre l'entente au quotidien. Weiss suggère des mesures très concrètes et pratiques pour chacune des phases. Ces conseils s'avèrent donc très utiles pour les personnes qui voudraient utiliser ce volume comme outil de formation et comme guide d'intervention.

Ainsi, pour la phase préparatoire, l'auteur souligne combien la composition d'une équipe de négociation envoie déjà un message important à l'autre partie sur le type de processus dans lequel elle veut s'engager. Le mandat qu'on définit à cette phase ne s'exprime plus en termes de résultats spécifiques à obtenir comme dans le cas de la négociation positionnelle, mais comme une position minimale ou une limite à partir de laquelle on a recours au pouvoir et aux méthodes traditionnelles. La connaissance de ce seuil permet non seulement à une partie d'évaluer les résultats obtenus jusque-là, mais lui fournit aussi un 
cadre de référence pour décider si elle a avantage ou non à retourner à l'ancien mode de négociation. Dans le cadre de la nouvelle approche, les revendications auparavant exprimées par des clauses spécifiques de convention collective sont plutôt formulées sous forme de problèmes à résoudre après qu'on ait identifié ensemble les intérêts communs et les intérêts divergents et que, par la suite, dans le cadre d'un remue-méninges, on va chercher des solutions mutuellement bénéfiques lors de la troisième phase. C'est lors de la phase suivante que les parties en arrivent à une entente sur la solution au problème. Les parties doivent toutefois s'assurer dès le départ de l'appui de leurs mandants les plus influents tant à l'égard du processus que des résultats qui en découlent au fur et à mesure des ententes qui se scellent. Pour Weiss, la cinquième phase est la plus difficile puisque les parties vont alors vivre la convention collective ; elles doivent comprendre que le règlement négocié ne représente pas un terme mais un commencement. Cette expérience sera cruciale pour le maintien, le développement ou la disparition du climat de confiance qui aura permis aux représentants patronaux et syndicaux de sortir du mode conflictuel.

Le dernier chapitre de l'ouvrage présente au lecteur sept pièges qui peuvent saboter la recherche de solutions mutuellement bénéfiques :

1. Une des parties résiste à la négociation basée sur les intérêts.

2. Elle se sert de la négociation basée sur les intérêts comme source de pouvoir, par exemple pour gagner du temps et retourner en mode conflictuel à un moment stratégiquement important qui lui permettra de remporter l'épreuve de force.

3. Négocier avec des amateurs représente une des pires difficultés car on ne peut savoir ce qu'ils vont faire puisqu'ils ne le savent pas euxmêmes !
4. Les deux parties veulent faire une seule équipe et tendent à oublier leurs différences.

5. Une partie a recours trop vite à ses vieilles habitudes, par exemple en se concentrant sur ses demandes plutôt que sur ses intérêts.

6. Manque de soutien de la part des hauts dirigeants.

7. Discordance dans les relations patronales-syndicales quotidiennes : le mode conflictuel au quotidien et le mode coopératif en négociation.

Weiss suggère des stratégies pratiques et éprouvées pour éviter ou surmonter ces pièges. Il ne laisse jamais entendre que l'implantation de ce mode de négociation est facile ct que les retours en arrière et les échecs sont impossibles. L'auteur reconnaît que certains enjeux se prêtent moins ou plus difficilement à la négociation basée sur les intérêts (ex., clauses salariales). Il reconnaît même qu'on peut négocier un ensemble de clauses sur le mode proposé alors que pour d'autres clauses on va retourner au mode plus positionnel. Même si l'auteur est un consultant pour qui ce type de négociation représente une source de revenus importante, le lecteur n'a jamais l'impression de lire une page publicitaire ou d'être exposé à un exercice de vente.

Comme le conclut Jean Boivin dans sa postface, le succès de la négociation basée sur les intérêts nécessite un contexte approprié. Les expériences indiquent que l'émergence de ce type de négociation peut provenir d'un sentiment de lassitude et de saturation à l'égard du rituel conflictuel, ou plus souvent, lors de bouleversements majeurs dans l'entreprise qui remettent en question les pratiques traditionnelles de relations du travail.

L'ouvrage de Weiss constitue donc une référence incontournable pour toute personne intéressée à comprendre le potentiel et les limites de la négociation 
basée sur les intérêts, et surtout à identifier les conditions et les stratégies pour en réussir l'implantation et le maintien.

MiChel GRANT

Département organisation et ressources humaines Université du Québec à Montréal

\section{The Discipline of Teamwork: Participation and Concertive Control}

by James R. BARKER, Thousand Oaks, Calif. : Sage Publications, 1999, 207 p., ISBN 0-7619-0369-0 (bond) and ISBN 0-7619-0370-4 (pbk).

James Barker's The Discipline of Teamwork makes a significant contribution to the already considerable literature on teams. In contrast to much of this literature, which focuses largely on how best to structure and implement teams for high performance, this work investigates teams as a mechanism for social control. Barker's book offers us a rare, critical look at the functioning of self managed teams, focusing in particular on how teams work to exert control over their members' behaviours. Barker recognizes explicitly that participation in such organizational forms has a price that workers are pressed to give more of their time and energy, to identify with the goals of the organization, and to collaborate effectively with their coworkers - all with obvious costs to their individual autonomy and personal lives.

The stated purpose of his book is threefold: to analyze how culture and control work in self-managed teams; to identify the consequences of a teambased culture; and to seek to change current thinking about these consequences so that teamwork can be made better for both the organization and for team members. The data upon which this study is based are drawn from the experiences of one firm: ISE Communications (a pseudonym), a manufacturer of electronic circuit boards used for voice and data transmission equipment. Barker gathered the data during three years of intensive field work in the early 1990s. During this time he was granted virtually unfettered access to management and workers at ISE, observing the manufacturing process, team meetings, more informal team interactions, and conducting hundreds of interviews with key informants.

Documenting the process by which the six self-managed teams at ISE came to develop their means of concertive control, Barker takes the reader inside teams to understand, in large part, why they are so successful - enabling management to do away with expensive supervision while ensuring the highly efficient production of high quality products. The heart of the book uses rich detail from Barker's conversations with informants and his observations from team interactions and meetings to document the process by which the teams adopted certain values; prioritized among competing values; converted their values into explicit, formalized rules; and then extracted compliance from members to those rules.

Barker shows through illustrative vignettes the power of the teams' control over their members. After early adoption of values that the teams believed would be functional for achieving the organization's objectives (such as good attendance, arriving to work and back from breaks on time, working for error free production, etc.), the teams quickly established specific and often writtendown guidelines or codes of conduct (such as, "if you come to work late three times and have made no effort to correct that problem, you will be let go"). These explicit rules were used to monitor and shape the behaviours of new team members who may not have had exposure to, and thus the opportunity to 